\title{
Monitoring the kinetic evolution of self-assembled SiGe islands grown by Ge surface thermal diffusion from a local source
}

\author{
G M Vanacore ${ }^{1,8}$, M Zani $^{1}$, M Bollani $^{2}$, E Bonera ${ }^{3}$, G Nicotra $^{4}, \mathbf{J ~ O s m o n d ~}^{5}$, \\ Giovanni Capellini ${ }^{6}$, Isella $^{7}$ and A Tagliaferri ${ }^{1}$ \\ ${ }^{1}$ CNISM-Dipartimento di Fisica, Politecnico di Milano, Piazza Leonardo da Vinci 32, I-20133 Milano, Italy \\ ${ }^{2}$ CNR-IFN, LNESS, Via Anzani 42, I-22100 Como, Italy \\ ${ }^{3}$ Dipartimento di Scienza dei Materiali and L-NESS, Università Milano-Bicocca, via Cozzi 53, I-20125 Milano, Italy \\ ${ }^{4}$ IMM-CNR, Stradale Primosole 50, I-95121 Catania, Italy \\ ${ }^{5}$ Institute of Photonics Sciences, E-08860 Barcelona, Spain \\ ${ }^{6}$ IHP, Im Technologiepark 25, 15236 Frankfurt (Oder), Germany \\ ${ }^{7}$ CNISM, LNESS, Dipartimento di Fisica, Politecnico di Milano (Polo Regionale di Como), Via Anzani 42, I-22100 \\ Como, Italy
}

E-mail: alberto.tagliaferri@ fisi.polimi.it

Received 10 October 2013, revised 30 December 2013

Accepted for publication 17 January 2014

Published 4 March 2014

\begin{abstract}
In this paper we experimentally study the growth of self-assembled $\mathrm{SiGe}$ islands formed on $\mathrm{Si}(001)$ by exploiting the thermally activated surface diffusion of $\mathrm{Ge}$ atoms from a local Ge source stripe in the temperature range $600-700{ }^{\circ} \mathrm{C}$. This new growth strategy allows us to vary continuously the Ge coverage from 8 to 0 monolayers as the distance from the source increases, and thus enables the investigation of the island growth over a wide range of dynamical regimes at the same time, providing a unique birds eye view of the factors governing the growth process and the dominant mechanism for the mass collection by a critical nucleus. Our results give experimental evidence that the nucleation process evolves within a diffusion limited regime. At a given annealing temperature, we find that the nucleation density depends only on the kinetics of the Ge surface diffusion resulting in a universal scaling distribution depending only on the Ge coverage. An analytical model is able to reproduce quantitatively the trend of the island density. Following the nucleation, the growth process appears to be driven mainly by short-range interactions between an island and the atoms diffusing within its vicinities. The islands volume distribution is, in fact, well described in the whole range of parameters by the Mulheran's capture zone model. The complex growth mechanism leads to a strong intermixing of $\mathrm{Si}$ and $\mathrm{Ge}$ within the island volume. Our growth strategy allows us to directly investigate the correlation between the Si incorporation and the Ge coverage in the same experimental conditions: higher intermixing is found for lower Ge coverage. This confirms that, besides the Ge gathering from the surface, also the Si incorporation from the substrate is driven by the diffusion kinetics, thus imposing a strict constraint on the initial Ge coverage, its diffusion properties and the final island volume
\end{abstract}

Keywords: SiGe islands, self-assembly, kinetics, surface diffusion, intermixing

\footnotetext{
8 Present address: Physical Biology Center for Ultrafast Science and Technology, Arthur Amos Noyes Laboratory of Chemical Physics, California Institute of Technology, 1200 East California Blvd, Pasadena, CA 91125, USA.
} 


\section{Introduction}

SiGe nanostructures have gained a large interest over the last years [1-4]. This attention has been driven mainly by the potential applications in micro, opto and nano-electronic devices $[5,6]$ thanks to the improvement of the optical and electronic properties compared to bulk systems [7], and by the high compatibility with existing silicon based technology. Besides the interest for applications, the $\mathrm{SiGe} / \mathrm{Si}(001)$ system is also a model for understanding the fundamental processes occurring during self-organization phenomena. In fact, the $\sim 4.2 \%$ lattice mismatch between $\mathrm{Ge}$ and $\mathrm{Si}$ introduces a hetero-epitaxial stress field. The stored elastic energy can then be partially relieved by spontaneous formation of 3D nanometric islands on top of a pseudomorphic wetting layer. This growth mode, called Stranski-Krastanov (SK) [8], has the potential to allow a low-cost and large-scale growth of self-assembled nanostructures, with applications in nanoelectronic devices exploiting the quantum confinement of charge carriers.

In recent years, considerable efforts have been devoted to the growth of hetero-epitaxial SiGe islands with well controlled shape [9, 10], size and position [11-14], and with defined stoichiometry and strain state [15-18]. Each of these objectives is at the same time a critical issue for both the fundamental understanding of the underlying physics and device engineering. The growth evolution has been widely investigated and several models have been proposed based on thermodynamic equilibrium and/or kinetics considerations, even though a complete and universally accepted picture is still a matter of controversy. The equilibrium shape of the islands is well described by a thermodynamic model [19], although the kinetic suppression of islands coalescence, leading to a uniform shape and narrow size distribution, has been recently reported [20,21]. Surface kinetics results in relevant mass transport effects, that play a fundamental role in the transition process between different island shapes [22, 23], and have also been shown to have a significant influence on the $\mathrm{Si}$ incorporation within a growing island [24,25]. Little is known about the dominant mechanism controlling the mass collected by a critical nucleus at the early stages of the growth process. Furthermore, it is still unclear whether the island growth is driven primarily by short-range interactions, affecting the diffusion over a length scale smaller than the average separation between the islands, or long-range interactions, extending over several island-to-island distances [26, 27]. Short-range interactions are controlled by ad-atom scattering and the atom binding energies at the island boundaries, which dominate within the area surrounding each island. On the other hand, non-local forces between the adatoms and the strain field generated by the islands within the substrate could promote the diffusion over distances greater than the average island separation and classify as long-range interactions.

In the present work, we investigate the growth of selfassembled $\mathrm{SiGe}$ islands on $\mathrm{Si}(001)$ obtained by surface thermal diffusion of Ge from a local solid state source. We monitor the dependence of the size, density and Ge content distributions for the obtained SiGe islands on the Ge coverage and on the annealing temperature in the range between 600 and 700

${ }^{\circ} \mathrm{C}$. We used lithographically defined Ge stripes as solid state sources fabricated on the Si surface. The stripes have been annealed to promote Ge atoms surface diffusion and island nucleation. With this new growth strategy we are able to create a continuously varying Ge coverage, and thus we can investigate the island growth over a wide range of dynamical regimes at the same time. Island growth methods with position-dependent growth rate and Ge coverage are attracting an increasing interest in the scientific community because of their unique potential, as proved by the very recent paper by Grydlik et al [28], almost simultaneous to our work, where the authors exploit the Ge surface diffusion from unpatterned to pit-patterned substrate regions. Although conceptually similar, our approach has the advantage of using a local solid state Ge source allowing for a direct external control of the growth and diffusion parameters, providing a unique approach to the determination of the factors governing the phases of nucleation and mass collection during the island growth.

We found that at high Ge coverage, the large flux of $\mathrm{Ge}$ atoms migrating on the surface tends to limit the $\mathrm{Si}$ incorporation from the substrate inducing the formation of a high density of small islands with a large Ge content. At low Ge coverages, the decrease of the local density of Ge atoms limits the number of supercritical nuclei and leaves a progressively longer time and a wider capture area for the $\mathrm{Si}$ incorporation leading to the formation of a low density of highly intermixed large islands.

At a given annealing temperature, the island density follows a universal scaling distribution depending only on the local Ge coverage. An analytical model based on the kinetics of diffusion is able to quantitatively reproduce the trend of the nucleation density. In this scenario the nucleation process mainly evolves within a diffusion limited regime. The volume distribution of the nucleated islands exhibits an inverse correlation with the density, and is well described in the whole range of parameters ( $\mathrm{Ge}$ coverages and annealing temperatures) by the Mulheran's capture zone model [29], according to which short-range interactions between the growing islands and the diffusing atoms in their capture area are the main driving forces in the competition between the islands to gather mass. The complex mechanism of the island growth leads to a strong intermixing of $\mathrm{Si}$ and $\mathrm{Ge}$ within the island volume, which is spatially correlated with the variation of the Ge coverage, and thus with the local density of $\mathrm{Ge}$ atoms (higher $\mathrm{Si}$ incorporation at lower Ge coverage). This confirms that, besides the Ge gathering from the surface, also the Si incorporation from the substrate is mainly driven by the diffusion kinetics, thus requiring that the final island volume depends only on the initial coverage of $\mathrm{Ge}$ and on its surface diffusion properties.

For the range of parameters investigated here, we demonstrate that energetic factors and non-local elastic fields play a minor role for the island growth. This rules out any effect that could create an unbalanced surface diffusion and an uneven exchange of atoms between the growing islands, and set the driving forces, the length and the time scales for the mass gathering and the final island configuration. 


\section{Experiment and methods}

Self-assembled SiGe islands are grown by surface thermal diffusion of Ge from solid state sources located directly on the $\mathrm{Si}$ surface. To this purpose we have fabricated pure Ge stripes, and annealed them in Ultra High Vacuum (UHV) at different temperatures in the range $600-700{ }^{\circ} \mathrm{C}$ inducing Ge diffusion and islands self-assembly. The Ge stripes (width $5 \mu \mathrm{m}$, length of several $\mathrm{mm}$ ) are produced by a photo-lithographic patterning of a pure Ge $150 \mathrm{~nm}$ thick film grown on a $\mathrm{Si}(001)$ substrate by low energy plasma enhanced chemical vapor deposition (LE-PECVD) [30]. The samples are annealed by direct Joule heating flowing a DC current through the Si substrate. A PHI 660 Scanning Auger Microscope (SAM) is used for in situ imaging and spatially resolved chemical analysis of the nucleated islands The average Ge content of single islands is obtained by a standard quantification method, where the Si LMM (90 eV) and Ge LMM (1150 eV) Auger line intensities measured on a single island are normalized by the values obtained for pure $\mathrm{Si}$ and $\mathrm{Ge}$ acquired in the same experimental conditions. Atomic force microscopy (AFM) for ex situ analysis of island density and volume distributions is performed using a Veeco Innova microscope operated in tapping mode with ultra-sharp tips (nominal tip radius about 2 $\mathrm{nm})$. Statistical analysis of AFM data is performed using free software tools [31]. The average composition of single islands is also measured by micro-Raman spectroscopy and cross-sectional Electron Energy Loss Spectroscopy (EELS) in a Scanning Transmission Electron Microscope (STEM). The Raman experiment is performed with a Jasco R800 double spectrometer using backscattering geometry and $458 \mathrm{~nm}$ as excitation wavelength focused through a 0.9 numerical aperture objective. The EELS-STEM experiment has been performed in cross-section geometry using a JEOL 2010F TEM/STEM equipped with a $200 \mathrm{kV}$ Schottky field emission gun. The Si and Ge L-edges loss signals (energy loss of $99 \mathrm{eV}$ and $1217 \mathrm{eV}$ with respect to the elastic peak, respectively) are measured as a function of the position along a vertical line scan on a single island. The relative content of $\mathrm{Si}$ and $\mathrm{Ge}$ was then derived from the integral intensities normalized to the Hartree-Slater cross-sections.

\section{Results and discussion}

During the high temperature annealing in UHV, the stripes act as sources from which Ge diffuses on the Si surface forming initially a continuous two-dimensional (2D) over-layer (OL). Using spatially resolved Auger analysis we measured the Ge LMM and Si LMM Auger lines along the diffusion profile as a function of the distance, $d$, from the stripe (in this case the electron beam is focused in regions in between the islands). The determination of the OL thickness has then been obtained by fitting the Auger line intensities with a discrete layer model [32], where the OL is approximated by a $\mathrm{Si}_{1-\alpha} \mathrm{Ge}_{\alpha}$ thin film of variable thickness and composition, $\alpha$. In the inset of figure 1(a) and in the figure 1(b) we report the obtained thickness and composition of such a layer for a typical case (annealing at $670{ }^{\circ} \mathrm{C}$ for $4.5 \mathrm{~min}$ ), where a gradient of the
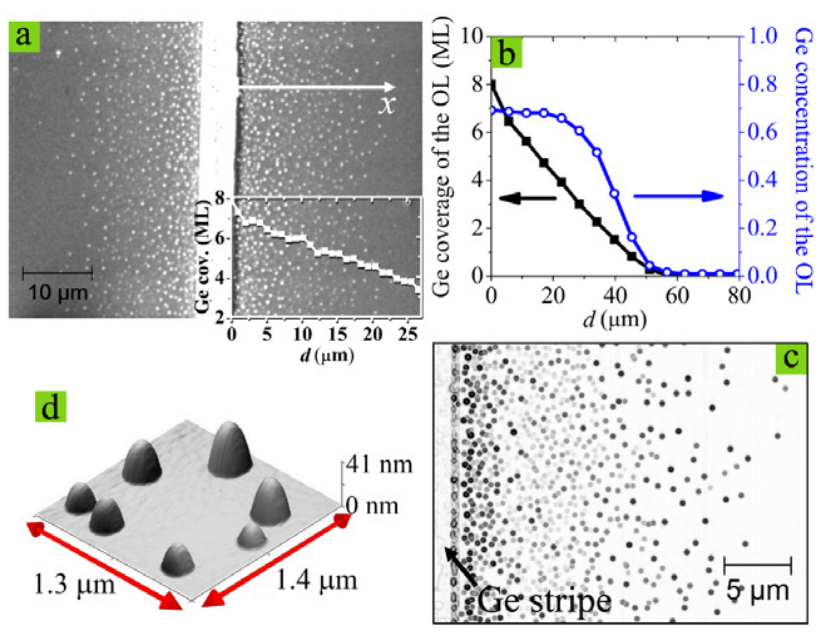

Figure 1. Ge surface diffusion and island nucleation after annealing at $670{ }^{\circ} \mathrm{C}$ for $4.5 \mathrm{~min}$. Panel (a) SEM image of the sample surface (the lighter part in the center is the Ge stripe); inset: Ge coverage as a function of the distance, $x$, from the stripe. Panel (b) Ge coverage and $\mathrm{Ge}$ fraction of the continuous over-layer (OL) created by the diffusive motion of $\mathrm{Ge}$ atoms from the stripe over the Si substrate. Panel (c) large area AFM image in gradient mode of the nucleation region (the stripe is on the left side of the image). Panel (d) 3D AFM images of self-assembled islands.

Ge coverage from 8 to $0 \mathrm{ML}$ is clearly seen. Typical values of the $\mathrm{Ge}$ composition for the continuous over-layer in the investigated temperature range are $\alpha=0.66$ (at $700{ }^{\circ} \mathrm{C}$ ) and 0.8 (at $600{ }^{\circ} \mathrm{C}$ ). The diffusion profile extends along the direction perpendicular to the stripe for a diffusion length, $L$, varying between a few $\mu \mathrm{m}$ to a few tens of $\mu \mathrm{m}$ according to the annealing time and temperature [32].

Spontaneous nucleation of self-assembled SiGe islands is observed to coexist with the continuous surface diffusion of Ge [33] as shown in figures 1(a) and (c)-(d) by scanning electron microscopy (SEM) and AFM images, respectively. It is clearly seen that the size and density of the nucleated islands vary as a function of the distance $d$ from the Ge stripe. Understanding the physical origin of this spatial variation, and of its dependence on temperature and Ge coverage, gives a fundamental insight into the factors governing the growth process.

\subsection{Microscopic evolution: scaling behavior of island growth}

In this section we investigate the wide-scale size distribution of the whole population of nucleated islands averaged over the varying Ge coverage on a length scale greater than $10 \mu \mathrm{m}$. Our results are then compared with the commonly accepted picture of microscopic growth evolution derived from deposition experiments (Molecular Beam Epitaxy, MBE, and Chemical Vapor Deposition, CVD).

Figure 2 shows the scatter plots of the island base area, $A_{\mathrm{b}}$, as a function of the volume, $V$, for the whole island population, for annealing at the two boundaries of the temperature range explored in this work $\left(600\right.$ and $\left.700{ }^{\circ} \mathrm{C}\right)$, and for islands in the region $d>2 \mu \mathrm{m}$ where re-incorporation in the Ge stripe is negligible as discussed below. At a fixed temperature, the islands exhibit a monotonically increasing dependence of 


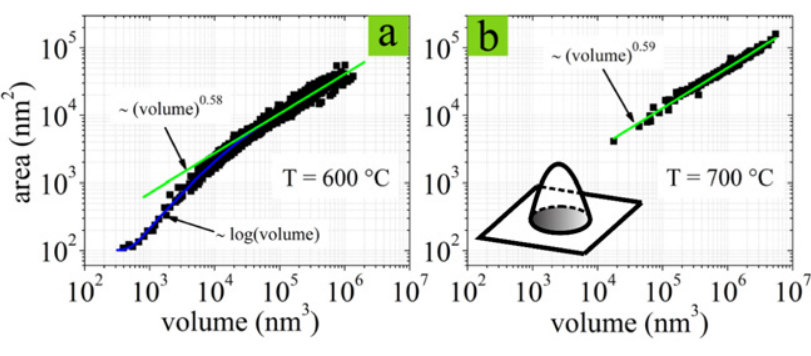

Figure 2. Scatter plots of the island base area as a function of the volume for the whole island population in the cases of annealing at $600{ }^{\circ} \mathrm{C}$ (panel (a)) and $700^{\circ} \mathrm{C}$ (panel (b)). The green lines in both panels represent a power-law behavior, while the blue curve in panel (a) is a logarithmic function (see text for details). Inset: schematic representation of a SiGe island; the gray shaded region represents the base area.

their base area with volume. Higher annealing temperatures lead to an increase of the mean island size. This behavior is reproducibly observed for different samples and for all the temperatures investigated. The obtained trend is very similar to that usually reported for island growth by deposition experiments as MBE [34] and CVD [35, 36], and reflects the basic thermally activated origin of the processes governing island growth (diffusion, nucleation and capture-see [37]).

Looking more quantitatively at the trend shown in figure 2 , we found that at high annealing temperatures $\left(700^{\circ} \mathrm{C}\right)$ the island base area scales with the volume according to a simple power law:

$$
A_{\mathrm{b}} \propto V^{\beta}
$$

where the exponent $\beta$ is equal to 0.59 (see figure 2(b)). This behavior is partially followed at lower annealing temperatures by the subset of larger volume islands, whose $\beta$ results to be $0.58\left(600{ }^{\circ} \mathrm{C}\right.$-see figure 2(a)). This monotonic dependence of the island base area on its volume can be described by the same mechanism proposed for island growth in deposition experiments: the evolution from small to large islands is obtained by a continuous transition through Ostwald ripening ${ }^{9}$, where the coarsening of larger islands at the expense of smaller ones dominates. At the high volume limit, the islands are thus characterized by a homogeneous (self-similar) growth, i.e. they increase their volume by increasing simultaneously both the area and the height whilst maintaining always the same global dome shape.

For the small volume islands, the global size distribution in figure 2(a) is no longer described by a power law, but indeed the island area scales with the volume by a slower logarithmic dependence $\left(A_{\mathrm{b}} \propto \log (V)\right)$, showing that small islands exhibits a preferential vertical growth, i.e. they increase in volume by increasing more rapidly in height rather than in base area. This trend can be also understood by recalling the results obtained by Montalenti et al [39] in a deposition

${ }^{9}$ The Ostwald ripening is the coarsening of the size distribution of an ensemble of islands driven by the Gibbs-Thomson effect (see Gibbs [38] and Thomson [38]). Within this framework an abrupt drop of the chemical potential occurs when the islands grow past a critical volume, triggering a coarsening process where the driving force depends on the mean curvature radius of the islands (see Chakraverty [38]). experiment. In that work the authors demonstrate that a shape transition from smaller to larger islands is mediated by a preferential accumulation of Ge atoms on the partially relaxed topmost region of the island, which represents an energetically favorable position. The global size distributions obtained in the case of island growth by surface thermal diffusion are thus consistent with those previously reported for deposition experiments, as MBE and CVD, proving that the mechanisms governing island formation are basically identical in both cases. However, although the wide-scale behavior is the same, the local size and density distributions (see below) exhibit intrinsic and peculiar properties due to the presence of a remote local Ge source and thus of a continuously varying Ge coverage.

\subsection{Density evolution: diffusion limited effects}

Using extensive AFM statistical analysis for each investigated sample, we have determined the behavior of the island density as a function of the distance, $d$, from the stripe (see figure 3 showing five cases at different annealing temperatures: 600, $625,650,670$ and $700{ }^{\circ} \mathrm{C}$ ). The density distributions present common features irrespective of the annealing temperature: the island density exhibit a maximum a few $\mu \mathrm{m}$ away from the stripe edge, and then slowly decreases at greater distances from it. This trend can be essentially described by the competition between two opposite processes. The first is the preferential nucleation at the stripe edge and the second one is the random nucleation on the flat $\mathrm{Si}$ surface, where the Ge coverage presents a gradient along the direction perpendicular to the stripe (1D geometry). As a result, Ge atoms moving on the surface can either meet each other to form islands, or migrate toward and be incorporated at the stripe edge. This competition leads to a depleted region, or 'denuded zone', in the spatial distribution of the islands beside the stripe [40, 41].

The final island density distribution is well reproduced by considering the two processes as statistically independent, resulting in a probability of island formation given by the product of the probability, $p_{1}$, that the atoms are not captured by the stripe edge, and the probability, $p_{2}$, of island nucleation on the flat Si surface. At a given temperature, the probability $p_{1}$ is minimal at the stripe edge and increases as the distance $d$ from the stripe increases, saturating at $d \sim 2-3 \mu \mathrm{m}$ [40](see red curve in figure 3(a)). However, in the present case the Ge coverage decreases moving away from the stripe. At distances greater than 2-3 $\mu \mathrm{m}$, where random nucleation on the flat $\mathrm{Si}$ surface dominates, the island density dependence is determined only by the probability of random nucleation $p_{2}$. This induces a decreasing island density as a function of the distance from the stripe, monotonically dependent on the decreasing local density of $\mathrm{Ge}$ atoms due to the diffusion from the stripe (see figure 1(b) and blue curve in figure 3(a)).

This interpretation is further supported by the temperature dependence of the island positional distribution: the island density decreases with the annealing temperature at a given Ge coverage (see figure 4(a)). This is genuinely a diffusion limited effect. In fact, the rate of Ge surface diffusion, and thus the diffusion length of each atom, exponentially increases 

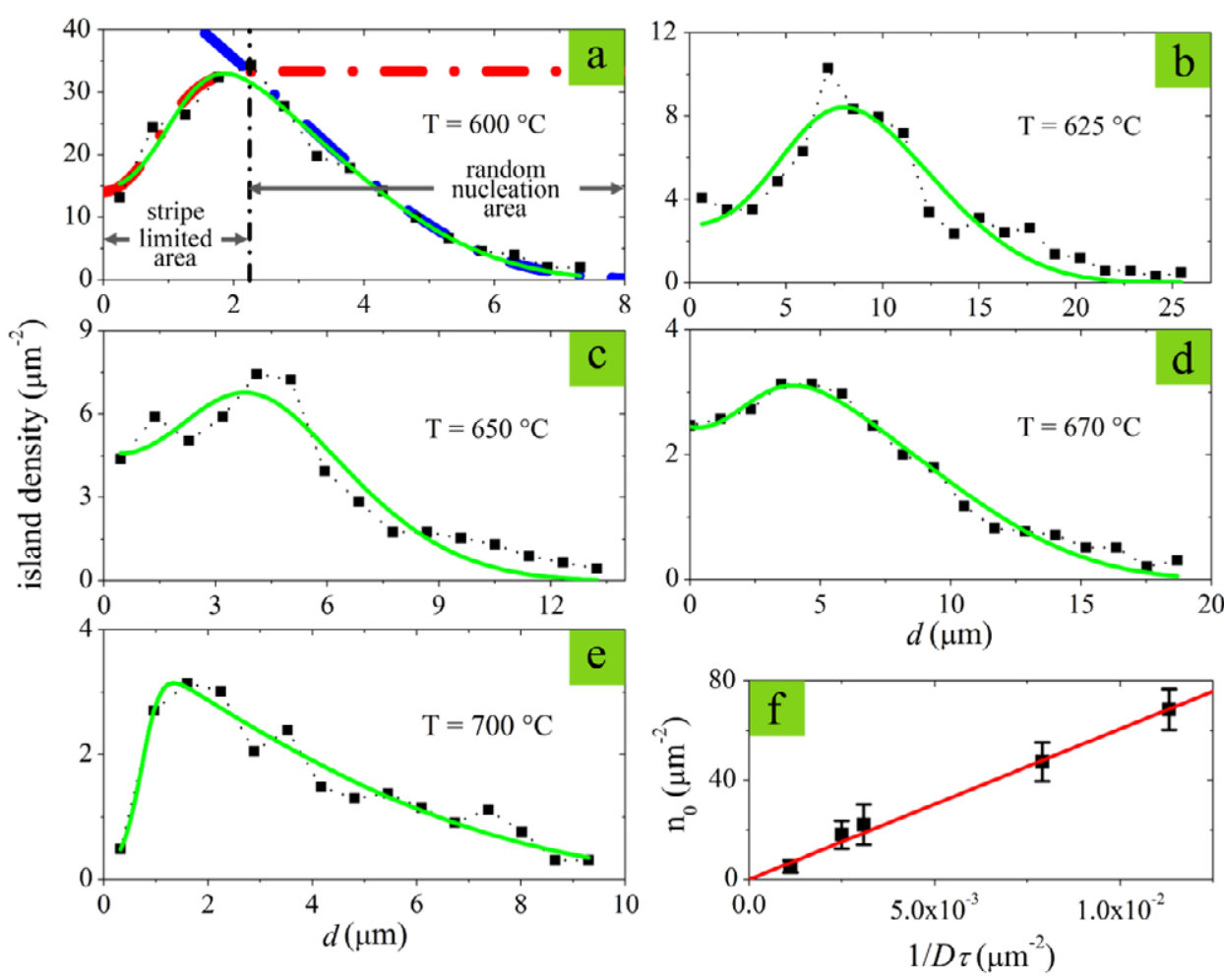

Figure 3. Panel (a)-(e) Experimental behavior (full black squares) of the island density as a function of the distance, $d$, from the stripe for annealing at $600{ }^{\circ} \mathrm{C}(\mathrm{a}), 625^{\circ} \mathrm{C}(\mathrm{b}), 650^{\circ} \mathrm{C}(\mathrm{c}), 670^{\circ} \mathrm{C}(\mathrm{d})$, and $700^{\circ} \mathrm{C}(\mathrm{e})$. The green curves are the best fitting of the experimental data using the $1 \mathrm{D}$ model of nucleation developed in this paper (see text for details). In panel (a), the red curve models the probability, $p_{1}$, that the atoms are not captured by the stripe edge, while the blue curve represents the probability, $p_{2}$, of island nucleation on the flat $\mathrm{Si}$ surface with a variable Ge coverage (see text for details). Panel (f) Linear scaling behavior exhibited by $n_{0}$ when plotted as a function of $1 / D \tau(D$ is the diffusion coefficient and $\tau$ is the annealing time).

with the temperature promoting the nucleation of islands at greater distance from each other, and thus leading to a smaller areal density [26].

Based on the above qualitative discussion, we developed a one-dimensional model of the mass transport and island nucleation that allows a complete quantitative description of the temperature and spatial dependencies of the island nucleation. We assume that the island density is mostly controlled by the local differences in the chemical potential $\Delta \mu$ of the wetting layer. Tersoff [42] used empirical potentials to calculate the surface energy per atom for Ge wetting layers as a function of thickness. Daruka and Barabasi [43] suggested an approximate exponential form for the change in the chemical potential with thickness:

$$
\Delta \mu=-\Delta \mu_{0} \exp \left(-\frac{h-h_{\mathrm{C}}}{h^{*}}\right)
$$

where $h$ is the wetting layer thickness, $h_{\mathrm{C}}$ is the critical WL thickness at the 2D-to-3D transition ( $\sim 4 \mathrm{ML}$ ), and $\Delta \mu_{0}$ and $h^{*}$ describes the wetting forces between Ge and Si [42, 43]. By fitting Tersoff's results (plotted as square symbols in figure 3 of [42]), we set $\Delta \mu_{0}=0.1 \mathrm{eV}$ and $h^{*}=a_{\mathrm{Ge}} / 4$ ( $a_{\mathrm{Ge}}$ is the Ge lattice parameter). Variation of the chemical potential of the islands as a result of a coarsening process can be neglected since it occurs on a time scale much smaller than the typical annealing time used here for our observations [22].
Therefore, in the nucleation model we have only considered the behavior of those critical nuclei energetically favorable with respect to the Ostwald ripening and whose stability and spatial configuration depend only on the local differences in the chemical potential of the wetting layer, $\Delta \mu$.

In our experiment the wetting layer thickness, $h$, is determined by the 1D Fick's second law, which describes the surface diffusion from a localized source. The Ge stripe has an initial concentration distribution with a step-like shape, and the height at the boundary is essentially maintained at a constant value during the annealing. These initial and boundary conditions represent the case of diffusion from a source of constant concentration. The solution of the diffusion equation, i.e. the $\mathrm{Ge}$ coverage along the diffusion profile is thus given by the following relation:

$$
h=h_{0}\left[1-\operatorname{erf}\left(\frac{d}{L}\right)\right]=h_{0} \operatorname{erfc}\left(\frac{d}{L}\right)
$$

where $L$ is the diffusion length. Equations (2) and (3) describe quantitatively the random nucleation on a flat $\mathrm{Si}$ surface where there exist a gradient for the Ge coverage along the direction perpendicular to the stripe. Now we need to introduce the preferential trapping at the stripe edge. This can be done by artificially creating at the stripe edge a Gaussian-shaped region of limited spatial extension exhibiting a local lowering of the chemical potential, as already used in [41] for the modeling 

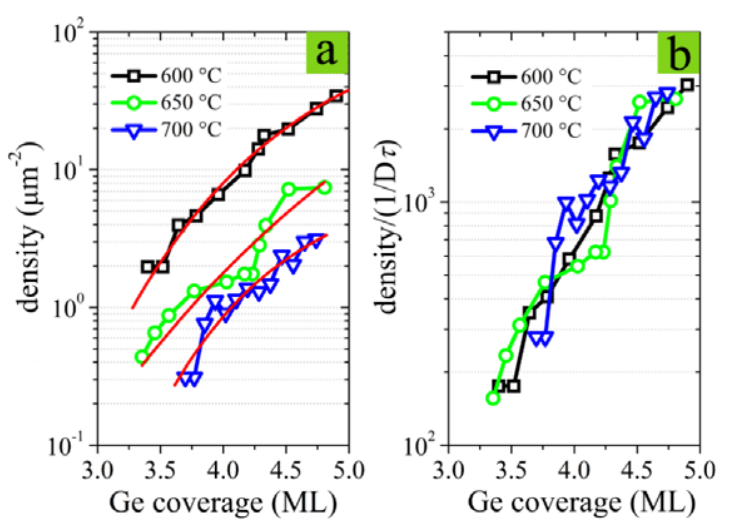

Figure 4. Panel (a) Island density in the random nucleation area $(d>2-3 \mu \mathrm{m})$ as a function of the Ge coverage for annealing at $600^{\circ} \mathrm{C}$ (black squares), $650^{\circ} \mathrm{C}$ (green circles), and $700^{\circ} \mathrm{C}$ (blue triangles). The red curves are the best fitting to the experimental data using the 1D model of nucleation developed in this paper. Panel (b) Island density normalized to the factor $1 / D \tau$. All curves follows a universal scaling distribution which depends only on $\mathrm{Ge}$ coverage.

of the denuded zones around dimples created in the substrate surface. The total chemical potential is thus given by:

$$
\begin{aligned}
\Delta \mu= & \Delta \mu_{1}+\Delta \mu_{2}=-\chi \exp \left(-\frac{d^{2}}{d_{0}^{2}}\right) \\
& -\Delta \mu_{0} \exp \left(-\frac{h-h_{\mathrm{C}}}{h^{*}}\right)
\end{aligned}
$$

where $\Delta \mu_{1}$ and $\Delta \mu_{2}$ are the contributions of the stripe edge and of the Ge coverage gradient, respectively, and $\chi$ the reduction of chemical potential at the stripe edge, centered in $d=0$, with $d_{0} / 2$ being approximately the width of the stripe edge.

Nucleation is then treated in our model by considering that for each surface site the relative probability, $p$, of formation of a critical nucleus depends only on the local differences in the chemical potential $\Delta \mu$ of the wetting layer. Since in the nucleation process a characteristic activation energy must be overcome, the probability $p$ follows an Arrhenius-type exponential law:

$$
\begin{aligned}
p & =p_{0} \exp \left(\frac{\Delta \mu}{k_{\mathrm{B}} T}\right) \\
& =p_{0} \exp \left(\frac{\Delta \mu_{1}}{k_{\mathrm{B}} T}\right) \exp \left(\frac{\Delta \mu_{2}}{k_{\mathrm{B}} T}\right)=p_{1} p_{2}
\end{aligned}
$$

where $\Delta \mu, \Delta \mu_{1}, \Delta \mu_{2}$ are given by the equation (4), $p_{0}$ is the probability of nucleation on a flat surface with homogeneous $\mathrm{Ge}$ coverage with no preferential nucleation sites and $p_{1}=\exp \left(\frac{\Delta \mu_{1}}{k_{\mathrm{B}} T}\right)$ and $p_{2}=p_{0} \exp \left(\frac{\Delta \mu_{2}}{k_{\mathrm{B}} T}\right)$ are the probabilities mentioned above. It is worth noticing that $\Delta \mu$ should become negative when the nucleus becomes supercritical and the island tends to increase its size. In this picture the island density, $n$, is determined by the number of supercritical nuclei, and it is thus proportional to the probability $p$ [44]:

$$
n=\frac{n_{0}}{p_{0}} p
$$

where $n_{0}$ represents the island density corresponding to the probability $p_{0}$.

By combining the equations (2)-(6), the dependence of the number of supercritical nuclei, i.e. the island density, on the distance from the source stripe can be finally obtained:

$$
\begin{aligned}
n= & n_{0} \exp \left[-\frac{\Delta \mu_{0}}{k_{\mathrm{B}} T} \exp \left(-\frac{h_{0} \operatorname{erfc}\left(\frac{d}{L}\right)-h_{\mathrm{C}}}{h^{*}}\right)\right. \\
& \left.-\frac{\chi}{k_{\mathrm{B}} T} \exp \left(-\frac{d^{2}}{d_{0}^{2}}\right)\right] .
\end{aligned}
$$

The green lines in figures 3(a)-(e) represent the best fitting of the experimental behavior of the island density as a function of $d$ using the analytical 1D model of nucleation discussed so far. All the important trends seen in the experiments are satisfactorily reproduced. The model has only two free fitting parameters, $n_{0}$ and $\chi$. In fact, $h_{0}$ and $L$ are determined by the experimentally measured diffusion profiles for each annealing temperature; $h_{\mathrm{C}}$ and $h^{*}$ are taken from literature as equal to 4 ML and $1 \mathrm{ML}$, respectively [42]; and the width of the stripe edge $d_{0} / 2$ is measured to be $\sim 0.5 \mu \mathrm{m}$ from AFM images. The $\chi$ values obtained from the fittings vary in the range $0.1-0.2 \mathrm{eV}$, very close to the value used for $\Delta \mu_{0}=0.1 \mathrm{eV}$ as derived by Tersoff's results, and similar to the value obtained in [41] where the island nucleation is studied around dimples created in the substrate surface. The heuristic character of the term describing the denuded zone in equation (4) leaves dormant the detailed information about its physical nature, which might deserve a better investigation in the future. However, this simple semi-empirical approach boasts a twofold achievement: (1) it demonstrate that the gross features of our experimental results can be described based on the kinetics of the diffusion alone, and (2) it allows to quantify the scaling dependence of the island density in the random diffusion area on the diffusion length, as discussed in the next paragraph.

Figure 3(f) shows the linear scaling behavior exhibited by $n_{0}$ when plotted as a function of $1 / D \tau$ ( $D$ is the diffusion coefficient and $\tau$ is the annealing time), where $D \tau$ is derived by the diffusion length $(L=2 \sqrt{D \tau})$ from the diffusion profiles measured at each annealing temperature [32]. This confirms quantitatively the diffusive origin of the decreasing gradient of the island density at higher temperatures. A further stringent support to this interpretation comes from the scaling analysis of the correlation between the island density within the random nucleation area $(d>2-3 \mu \mathrm{m})$ and the Ge coverage (see figure 4(a)). Normalizing the island density to the factor $1 / D \tau$, we found that all curves follow a universal scaling distribution which depends only on the Ge coverage (see figure 4(b)), where the number of islands in a $L^{2}$ area changes of about one order of magnitude for Ge coverages changing from 3.5 to $5 \mathrm{ML}$. This can be easily understood assuming that the growth process evolves within a diffusion limited regime. In this case the island density can be written as the inverse of the squared distance $d_{\mathrm{nn}}^{2}$ between nearest-neighbor islands, which scales as the diffusion length $L$ of $\mathrm{Ge}$ atoms moving on the surface. In the hypothesis of a random walk motion, $L$ is related to the diffusion coefficient and the annealing time by the relation 

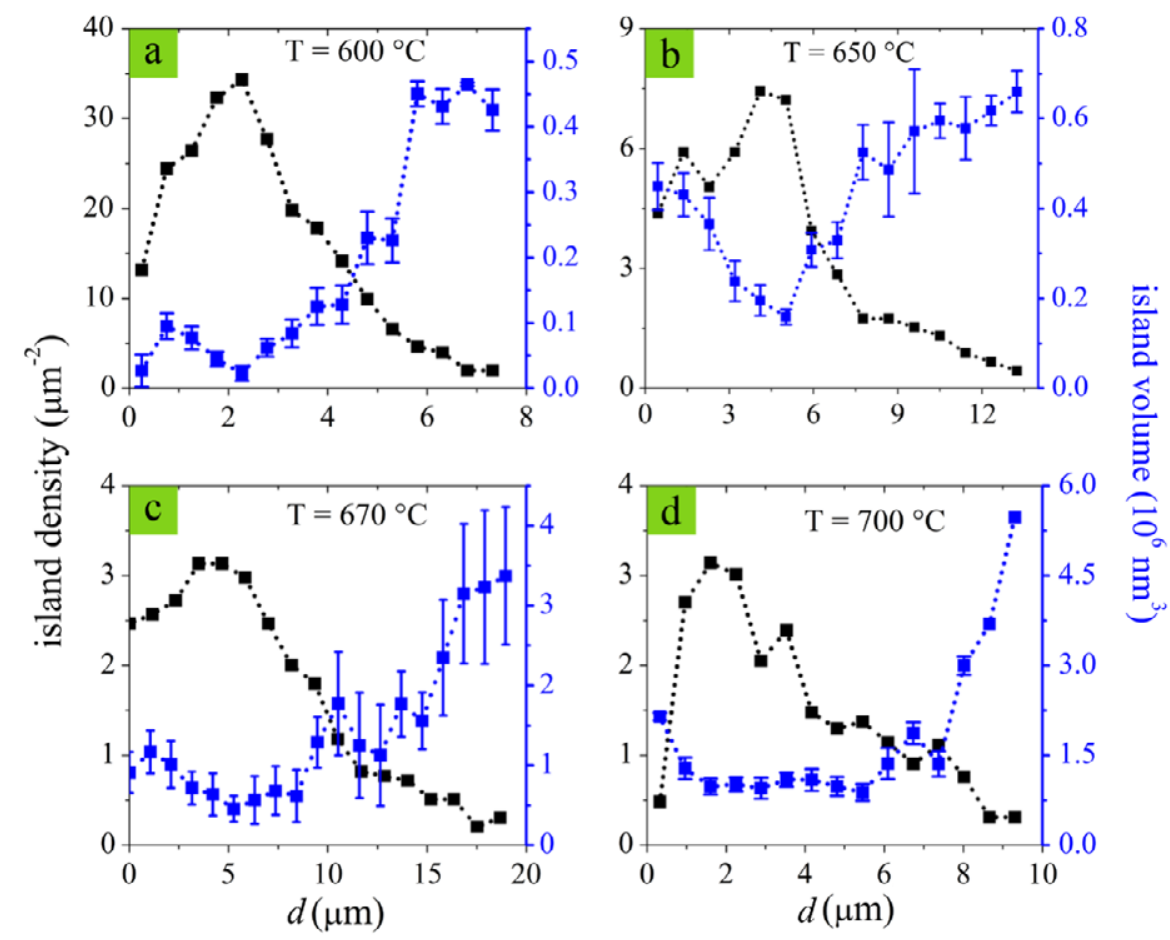

Figure 5. Experimentally observed volume evolution (blue squares) of the islands as a function of distance, $d$, from the stripe for different annealing temperatures: $600^{\circ} \mathrm{C}(\mathrm{a}), 650^{\circ} \mathrm{C}(\mathrm{b}), 670^{\circ} \mathrm{C}$ (c) and $700^{\circ} \mathrm{C}(\mathrm{d})$. The volume trend is shown in comparison with the density distributions (black squares).

$L^{2}=4 D \tau$. This leads to the following expression for the island density:

$$
n \approx \frac{1}{d_{\mathrm{nn}}^{2}} \approx \frac{1}{L^{2}} \approx \frac{1}{D \tau}
$$

confirming the experimentally observed scaling behavior.

Although it is reasonable that the island density depends on the local Ge ad-atom density, here we demonstrate that the ad-atom density is the only factor determining the island density through the scaling behavior shown in figure $4(\mathrm{~b})$, which implies a more strict relation between the island growth and the diffusion properties.

\subsection{Size evolution: capture zone growth}

In figure 5 the size evolution of the islands as a function of distance, $d$, from the stripe is shown and compared with their density: the region with high Ge coverage (close to the stripe, $d \approx 0 \div 2-3 \mu \mathrm{m}$ ) presents the maximum island density and the lowest average island size, while where the coverage decreases to 3.5-4 ML larger average dimensions and lower island density are attained. The interplay among nucleation, atomic diffusion dynamics and Si intermixing can be strongly affected by the gradient of the Ge coverage as induced by the mass transport from the source stripe, and have a dominant role on the observed spatial modulation of island size.

The understanding of the origin of this size modulation can give a fundamental insight within the comprehension of the factors governing the relative growth of individual islands. Different competitive pathways have been proposed so far in literature in order to describe the growth evolution of SiGe islands and the gathering of the available mass on the surface among the different nuclei. Upon formation, the critical nucleus starts capturing the diffusing atoms in its neighborhood [44]. From this point on some different scenarios could develop during the further growth of the single nucleus. On one hand, the growth of the islands might be governed by diffusive phenomena [45] and by short-range interactions between the growing island and the diffusing atoms. In this framework, the diffusing atoms would tend towards and might be captured by the closest nucleus. The process could then be described by the Mulheran's capture zone model [29, 46], which results in a linear relationship between the growth rate of every island and the capture zone from which islands can gather mass. Accordingly, the competition among neighboring nuclei to attract the mass supplied to the surface would follow local laws and be mediated by short-range interactions. On the other hand, a growth process not consistent with the Mulheran's model could arise from long-range interactions, such as local non-uniformities in the mass density induced by elastic interactions among the islands, able to drive a preferential diffusion of atoms over length scales of several island nearest-neighbor distances [47]. In this case, the geometric proximity would not be the only parameter defining the capture zone, which would then no longer linearly related to the growth rate. Here we test these concepts for the island growth by surface thermal diffusion by comparing our experimental results with an ideal ensemble of islands whose growth is described by the capture zone model.

According to the Mulheran's model, the island volume, $V$, is proportional to the capture zone area, $A_{\mathrm{CZ}}$, and the island 

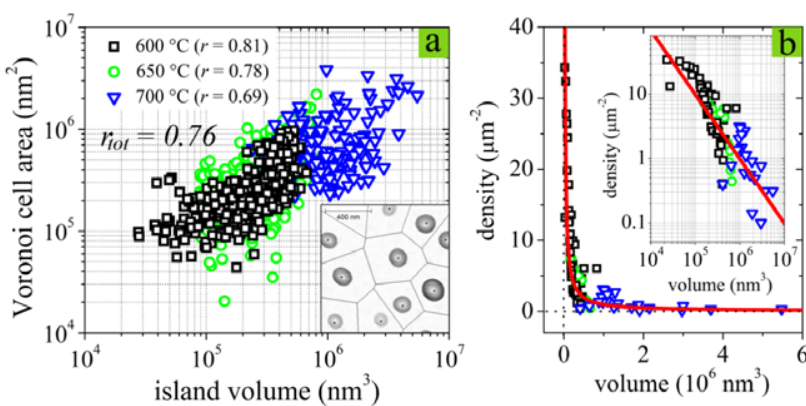

Figure 6. Panel (a) Scatter plot of the Voronoi cell area as a function of the island volume for islands grown inside the random nucleation area far from the stripe $(d>2 \mu \mathrm{m})$, and for annealing at $600^{\circ} \mathrm{C}$ (black squares), $650^{\circ} \mathrm{C}$ (green circles), and $700^{\circ} \mathrm{C}$ (blue triangles). The degree of linear correlation is estimated by calculating the Pearson's coefficient, $r$. Inset: schematics of the Voronoi tessellation of the island network reproduced on a representative AFM image in gradient mode. Panel (b) Island density as a function of the island volume. The red curve represents a rectangular hyperbola function $(n \approx 1 / V)$. Inset: $\log -\log$ plot of the data and the hyperbola represented in the main panel.

density, $n$, exhibits an inverse correlation with it:

$$
V \propto A_{\mathrm{CZ}}, \quad n \propto \frac{1}{A_{\mathrm{CZ}}} .
$$

The capture zone area can be defined quantitatively as the cell area of the Voronoi tessellation of the island network (see inset in figure $6(\mathrm{a}))^{10}$. The model's validity can be verified by evaluating the degree of linear correlation between island volumes and the Voronoi cell areas, quantified by the Pearson's coefficient, $r$ [44]. Figure 6(a) summarizes the results we obtained at different temperatures for islands grown inside the random nucleation area far from the stripe. An overall Pearson's coefficient of about 0.76, including results from all temperatures, attests the good linear correlation between the volume of the islands and their correspondent capture areas. The inverse correlation of the island volume as a function of the areal density $(n \approx 1 / V)$ shown in figure $6(\mathrm{~b})$ is an equivalent test of the same correlation, as from equation (9). Thus our results give experimental evidence that the Mulheran's model can satisfactorily predict the final island volumes in the whole investigated range of $\mathrm{Ge}$ coverages and diffusion temperatures. It is worth noting that we did not address the issue of the strain state of the islands since the model does not take into account any lattice deformation. The observed agreement between experimental results and model confirms that this hypothesis is consistent.

The identification of the relative importance between local and non-local interactions for the mass gathering thus allows describing the driving forces, the length and time scales for the ad-atoms to preferentially reach the islands and the final island configuration.

${ }^{10}$ The Voronoi tessellation is obtained by dividing the surface into several cells associated with the center of mass of each island and defined as the sets of points that are closer to a given island rather than to any other.

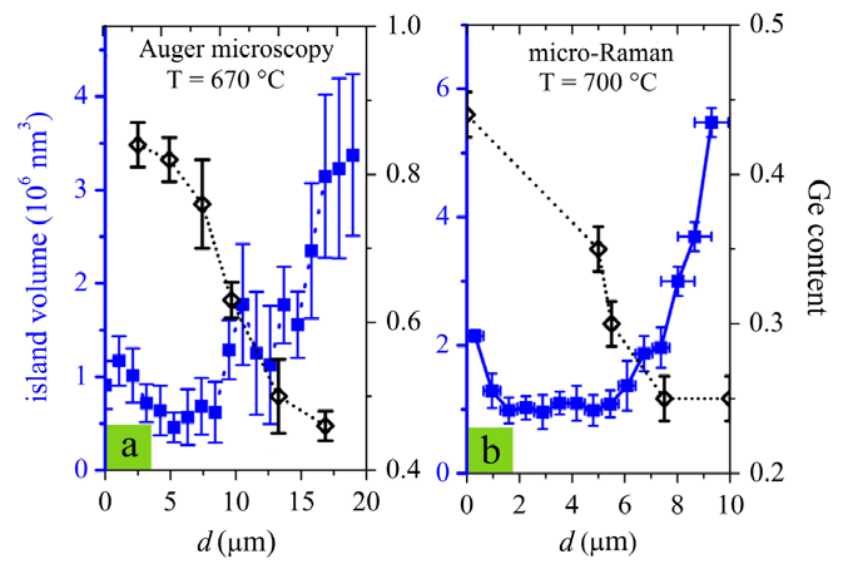

Figure 7. Panel (a) Trend of the Ge content, $x$, in single islands grown at $670{ }^{\circ} \mathrm{C}$ measured by scanning Auger microscopy (black diamonds) as a function of the distance, $d$, from the stripe edge. For comparison the volume distribution (blue squares) is also shown.

Panel (b) Trend of the Ge content in single islands grown at $700{ }^{\circ} \mathrm{C}$ measured by micro-Raman (black diamonds) as a function of the distance, $d$, from the stripe edge. For comparison the volume distribution (blue squares) is also shown.

\subsection{Ge content evolution: SiGe intermixing}

In this section we investigate the dependence of the Ge content within the island volume on the Ge coverage. It is well known that for $\mathrm{Si}_{1-x} \mathrm{Ge}_{x}$ islands on $\mathrm{Si}$ substrates the mass available for a growing island includes both $\mathrm{Ge}$ atoms, diffusing on the surface, and $\mathrm{Si}$ atoms, coming from the substrate. As widely demonstrated by many studies using deposition experiments, $\mathrm{Si}$ penetrates into the islands, as driven by the entropy of mixing [48] and strain relaxation, leading to the formation of alloyed nanocrystals and inducing an increase of the mean island size $[15,35,49,50]$.

To explore the interplay between Ge coverage, intermixing and size evolution for islands grown by surface thermal diffusion, we measured the composition of single islands as a function of the distance, $d$ from the stripe by means of scanning Auger microscopy, micro-Raman spectroscopy and EELS-STEM.

Figure 7(a) shows the trend of the Ge content monitored by SAM for islands grown at $670{ }^{\circ} \mathrm{C}$. For comparison the volume distribution is also shown. It is worth noting that the Auger analysis is sensitive only to the surface composition of the islands (its depth sensitivity is a few $\mathrm{nm}$ ) mediated over the lateral beam spot size $(\sim 100-300 \mathrm{~nm})$. This means that the composition values presented in figure 7 (a) are relative to the most external shell of the island, which is nevertheless significantly correlated to the mean Ge content [51]. This is confirmed by the cross-sectional composition analysis performed by EELS-STEM (see figure 8), showing a vertical compositional gradient of the Ge content from $x=0.55$ at the bottom to $x=0.78$ at the top of an island nucleated at $670{ }^{\circ} \mathrm{C}$ around $2 \mu \mathrm{m}$ away from the stripe edge, which is very close to the value of $x=0.84$ measured by Auger microscopy. Looking at the composition trend obtained by the SAM data as a function of $d$ in figure 7(a), a greater $\mathrm{Si}$ incorporation is found in the skin layer of larger islands nucleated farther 


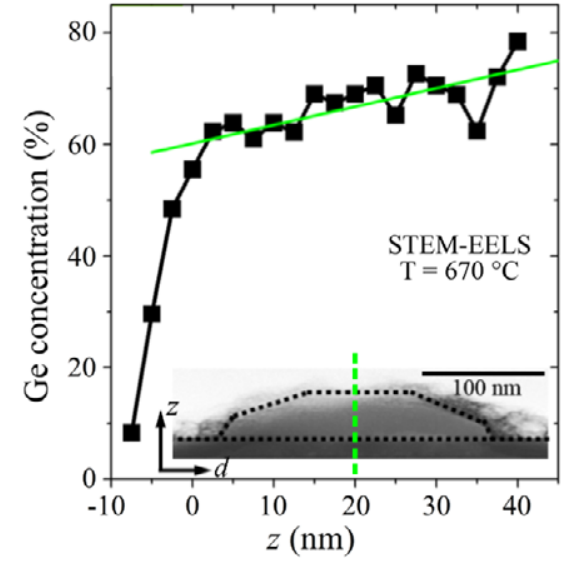

Figure 8. Cross-sectional composition analysis performed by EELS-STEM on a single island nucleated at $670{ }^{\circ} \mathrm{C}$ around $2 \mu \mathrm{m}$ away from the stripe edge. The cross-section profile shows a vertical compositional gradient of the Ge concentration from $55 \%$ at the bottom to $78 \%$ at the top.

away from the stripe in the regions with a lower Ge coverage. This behavior is also confirmed by micro-Raman spectroscopy, which is able to provide the island Ge content averaged over the whole island volume, since the laser beam penetrates deeper into the sample with respect to the electron beam. The value of Ge content for each island is obtained by fitting the measured Raman frequencies for the $\mathrm{Si}-\mathrm{Si}, \mathrm{Si}-\mathrm{Ge}$ and $\mathrm{Ge}-\mathrm{Ge}$ phonon modes with the model proposed in [52]. In figure $7(\mathrm{~b})$, the $\mathrm{Ge}$ composition monitored by micro-Raman is shown for islands grown at $700{ }^{\circ} \mathrm{C}$ as a function of the distance, $d$, from the stripe edge. For comparison the volume distribution is also shown. The measured value of Ge content changes from $x=45 \%$ to about $x=25 \%$ moving farther away from the stripe, with an experimental uncertainty of $\sim 3 \%$. The observation of a neat variation of composition as a function of the distance from the stripe is well distinguished from the experimental uncertainty and is in agreement with the SAM data.

The modulation of the island composition is thus spatially correlated with the variation of Ge coverage as induced by the mass transport from the stripe. In fact, close to the stripe edge the high Ge flux kinetically limits the $\mathrm{Si}$ incorporation within the growing islands, while away from the stripe the progressive decrease of the local density of Ge atoms leaves a progressively longer time and a wider capture area for the Si incorporation from the substrate This means that, besides the Ge gathering from the surface, also the $\mathrm{Si}$ incorporation from the substrate is mainly driven by the diffusion kinetics and thus can be well described within the capture zone picture presented in the previous section In our case of island growth by surface thermal diffusion, the validity of the Mulheran's model over the large range of parameters explored (Ge coverage and temperature), able to include also the strong island composition variability, supports the scenario where diffusion kinetics and short-range interactions are the main driving forces in the competition between the islands to gather their mass ( $\mathrm{Si}$ and $\mathrm{Ge}$ ) in the temperature range between 600 and $700{ }^{\circ} \mathrm{C}$. Thus the final island volume results proportional to the coverage and the diffusion length of $\mathrm{Ge}$ :

$$
\frac{V}{L^{2}} \propto \frac{1}{n L^{2}} \propto f(\text { Ge coverage }),
$$

and thus:

$$
V \propto L^{2} f(\text { Ge coverage })
$$

where the universal function $n L^{2}$ of the Ge coverage represented in figure 4(b) has been introduced in equations (10) and (11).

\section{Conclusions}

In this paper we experimentally explore the growth of selfassembled SiGe islands formed by thermally activated surface diffusion of $\mathrm{Ge}$ on $\mathrm{Si}(001)$ from a spatially defined Ge source. We monitor the dependence of the size, density and Ge content distributions for the obtained $\mathrm{SiGe}$ islands on the Ge coverage and on the annealing temperature in the range between 600 and $700{ }^{\circ} \mathrm{C}$. With this new growth strategy we are able to create a continuously varying Ge coverage (from 8 to $0 \mathrm{ML}$ ), and thus we can investigate the growth process over a wide range of dynamical regimes at the same time, providing a complete description of the growth evolution.

Our results give experimental evidence that the nucleation process evolves within a diffusion limited regime, where the island density follows a universal scaling distribution depending only on the Ge coverage. The island nucleation has been successfully reproduced by an analytical one-dimensional model, where the probability of formation of a critical nucleus depends only on the local differences in the chemical potential of the continuous over-layer. Once the island is nucleated, its final size is mainly determined by the kinetics of surface diffusion and by the short-range interactions between the growing islands and the diffusing atoms within the island capture area. These interactions set the driving forces, the length and time scales for gathering mass. In fact, the Mulheran's capture zone model has been shown to predict the final island volumes in the whole investigated range of Ge coverages and diffusion temperatures, with a Pearson's correlation coefficient of 0.76 between the island volume and the capture areas. The complex mechanism of the $\mathrm{SiGe}$ island growth leads to a strong intermixing of $\mathrm{Si}$ and $\mathrm{Ge}$ within the island volume, which is spatially correlated with the variation of the Ge coverage, and thus with the local density of $\mathrm{Ge}$ atoms (higher $\mathrm{Si}$ incorporation at lower Ge coverage). This confirms that, besides the Ge gathering from the surface, also the $\mathrm{Si}$ incorporation from the substrate is mainly driven by the diffusion kinetics, thus requiring that the final island volume depends only on the initial coverage of $\mathrm{Ge}$ and on its surface diffusion properties

\section{Acknowledgments}

This work was mainly supported by the CARIPLO Foundation through the EIDOS 2011-0382 project. A partial support by the Italian Ministry of Research MIUR through grant No. PRIN-20094W2LAY is also acknowledged. 


\section{References}

[1] Voigtänder B 2001 Surf. Sci. Rep. 43127

[2] Brunner K 2002 Rep. Prog. Phys. 6527

[3] Berbezier I and Ronda A 2009 Surf. Sci. Rep. 6447

[4] Aqua J-N, Berbezier I, Favre L, Frisch T and Ronda A 2013 Phys. Rep. 52259

[5] Kar G S, Kiravittaya S, Denker U, Nguyen B Y and Schmidt O G 2006 Appl. Phys. Lett. 88253108

[6] Katsaros G et al 2010 Nature Nanotechnol. 5458

[7] Petroff P M, Lorke A and Imamoglu A 2001 Phys. Today 5446

[8] Mo Y W, Savage D E, Swartzentruber B S and Lagally M G 1990 Phys. Rev. Lett. 651020

[9] Chen G, Lichtenberger H, Bauer G, Jantsch W and Schäffler F 2006 Phys. Rev. B 74035302

[10] Stoffel M, Rastelli A, Stangl J, Merdzhanova T, Bauer G and Schmidt O G 2007 Phys. Rev. B 75113307

[11] Capellini G, De Seta M, Evangelisti F, Zinovyev V A, Vastola G, Montalenti F and Miglio L 2006 Phys. Rev. Lett. 96106102

[12] Bollani M, Chrastina D, Fedorov A, Sordan R, Picco A and Bonera E 2010 Nanotechnology 21475302

[13] Zhong Z, Schwinger W, Schaffler F, Bauer G, Vastola G, Montalenti F and Miglio L 2007 Phys. Rev. Lett. 98176102

[14] Zhang J J, Rastelli A, Groiss H, Tersoff J, Schaeffler F, Schmidt O G and Bauer G 2009 Appl. Phys. Lett. 95183102

[15] De Seta M, Capellini G and Evangelisti F 2008 Phys. Rev. B $\quad 77045431$

[16] Gatti R, Marzegalli A, Zinovyev V A, Montalenti F and Miglio L 2008 Phys. Rev. B 78184104

[17] Schulli T U et al 2009 Phys. Rev. Lett. 102025502

[18] Bollani M et al 2012 Nanotechnology 23045302

[19] Medeiros-Ribeiro G, Bratkovski A M, Kamins T I, Ohlberg D A A and Williams R S 1998 Science 279353

[20] McKay M R, Venables J A and Drucker J 2008 Phys. Rev. Lett. 101216104

[21] McKay M R, Venables J A and Drucker J 2009 Solid State Commun. 1491403

[22] Ross F M, Tersoff J and Tromp R M 1998 Phys. Rev. Lett. 80984

[23] Rastelli A, Stoffel M, Tersoff J, Kar G S and Schmidt O G 2005 Phys. Rev. Lett. 95026103

[24] Malachias A, Stoffel M, Schmidbauer M, Schulli T U, Medeiros-Ribeiro G, Schmidt O G, Magalhães-Paniago R and Metzger T H 2010 Phys. Rev. B 82035307

[25] Vastola G, Shenoy V B, Guo J and Zhang Y-W 2011 Phys. Rev. B 84035432

[26] Aqua J-N and Frisch T 2008 Phys. Rev. B 78121305
[27] Grima R, DeGraffenreid J and Venables J A 2007 Phys. Rev. B 76233405

[28] Grydlik M, Brehm M, Hackl F, Schaffler F, Bauer G and Fromherz T 2009 Phys. Rev. B 88115311

[29] Mulheran P A and Blackman J A 1996 Phys. Rev. B 5310261

[30] Isella G, Chrastina D, Rössner B, Hackbarth T, Herzog H-J, König U and von Känel H 2004 Solid-State Electron. 481317

[31] Horcas I et al 2007 Rev. Sci. Instrum. 78013705 Gwyddion http://gwyddion.net/

[32] Vanacore G M, Zani M, Isella G, Osmond J, Bollani M and Tagliaferri A 2010 Phys. Rev. B 82125456

[33] Vanacore G M, Zani M, Bollani M, Colombo D, Isella G, Osmond J, Sordan R and Tagliaferri A 2010 Nano Res. Lett. 51921

[34] Katsaros G et al 2005 Phys. Rev. B 72195320

[35] Capellini G, De Seta M and Evangelisti F 2001 Appl. Phys. Lett. 78303

[36] Leite M S, Kamins T I and Medeiros-Ribeiro G 2009 Appl. Phys. Lett. 94053118

[37] Fanfoni F and Tomellini M 2005 J. Phys.: Condens. Matter 17 R571

[38] Thomson W 1871 Phil. Mag. Ser. 42448 Gibbs J W 1876 Trans. Connect. Acad. Sci. 3108 Chakraverty B K 1967 J. Phys. Chem. Solids 282401 Chakraverty B K 1967 J. Phys. Chem. Solids 282413

[39] Montalenti F et al 2004 Phys. Rev. Lett. 93216102

[40] Kim H J, Zhao Z M, Liu J, Ozolins V, Chang J Y and Xie Y H 2004 J. Appl. Phys. 956065

[41] Schwarz-Selinger T, Foo Y L, Cahill D G and Greene J E 2002 Phys. Rev. B 65125317

[42] Tersoff J 1991 Phys. Rev. B 439377

[43] Daruka I and Barabasi A-L 1998 Appl. Phys. Lett. 722102

[44] Ratto F, Locatelli A, Fontana S, Kharrazi S, Ashtaputre S, Kulkarni S K, Heun S and Rosei F 2006 Phys. Rev. Lett. 96096103

[45] Brinkmann M, Graaf S and Biscarini F 2002 Phys. Rev.

B $\quad 66165430$

[46] Pratontep S, Brinkmann M, Nuesch F and Zuppiroli L 2004 Phys. Rev. B 69165201

[47] Raiteri P and Miglio L 2002 Phys. Rev. B 66235408

[48] Medeiros-Ribeiro G and Stanley Williams R 2007 Nano Lett. 7223

[49] Tersoff J 1998 Phys. Rev. Lett. 813183

[50] De Seta M, Capellini G, Evangelisti F and Spinella C 2002 J. Appl. Phys. 92614

[51] Rastelli A, Stoffel M, Malachias A, Merdzhanova T, Katsaros G, Kern K, Metzger T H and Schmidt O G 2008 Nano Lett. 81404

[52] Pezzoli F et al 2008 Mater. Sci. Semicond. Process. 11279 